\title{
Presence of bilirubin in bronchobiliary fistula easily confirmed with urinary dipstick test and treated with embolization
}

\author{
Min Je Kim, Seon Hye Kim, In Kyoung Hwang, Hyung Oh Kim, Na Eun Jang, Seung Sook Paik, \\ and Myung Jae Park
}

Department of Pulmonary and Critical Care Medicine, Kyung Hee University College of Medicine, Seoul, Korea
Received: June 25, 2015

Revised : July 20, 2015

Accepted: July 22, 2015

\section{Correspondence to}

Myung Jae Park, M.D.

Department of Pulmonary and Critical Care Medicine, Kyung Hee University College of

Medicine, 23 Kyungheedae-ro,

Dongdaemun-gu, Seoul 02447,

Korea

Tel: +82-2-958-8197

Fax: +82-2-968-1848

E-mail:mjpwis@chol.com
To the Editor,

Bronchobiliary fistula (BBF) is defined as abnormal communication between the biliary tract and the bronchial tree. Biloptysis (the coughing up of bile) is pathognomonic of BBF, but patients can also have other respiratory symptoms such as chronic cough and dyspnea [1]. BBF usually occurs either in the congenital form, or following thoracoabdominal trauma or hepatic hydatid infection. However, BBF can also occur as a rare complication of biliary tract disease. BBF is difficult to prevent and its treatment is associated with high morbidity and mortality. Here we report an unusual case of a 53-year-old man with acquired, nontraumatic BBF due to complication of biliary tract disease. His BBF was diagnosed using a urine dipstick test and successfully treated with lipiodol embolization.

A 53-year-old male visited hospital presenting with fever, cough, and brownish sputum that had persisted for 2 months. He had been previously diagnosed with liver cirrhosis and as a hepatitis B carrier. In 2013, he was also diagnosed with hepatocellular carcinoma. On March 2013 and February 2014, two separate instances of transcatheter arterial chemoembolization (TACE) were performed. However, after the second TACE session, multiple hepatic abscesses and biloma developed due to bile duct injury. The abscesses were promptly drained and a percutaneous transhepatic biliary drainage (PTBD) tube was inserted. The post-procedure course was uneventful, and he was discharged 30 days after the procedure.

He remained well for 4 months, after which he developed brownish sputum (Fig. 1), a cough, and fever. At his second admission on September 2014, he appeared acutely ill and his general state was poor. On initial assessment, his temperature was $37.7^{\circ} \mathrm{C}$; however, he was hemodynamically stable. Physical examination did not reveal any abnormalities, with the exception of light tenderness in the right upper quadrant. No organs were palpable and the abdomen was soft. Biochemical analyses revealed leukocytosis, neutrophilia, and anemia. His biochemical results were white blood cell count, 10,700/ $\mathrm{mm}^{3}$ (segmented neutrophils, 88.2\%); hemoglobin, 8.9 $\mathrm{g} / \mathrm{dL}$; and platelet count, $204,000 / \mathrm{mm}^{3}$. An acute phase response was evident, with C-reactive protein at $10 \mathrm{mg} / \mathrm{dL}$. A cholestatic profile of hepatic enzymes was also observed (alkaline phosphatase, $495 \mathrm{IU} / \mathrm{L} ; \boldsymbol{\gamma}$-glutamyl-transferase, 261 U/L). Chest X-ray scans demonstrated peribronchial infiltration in the 


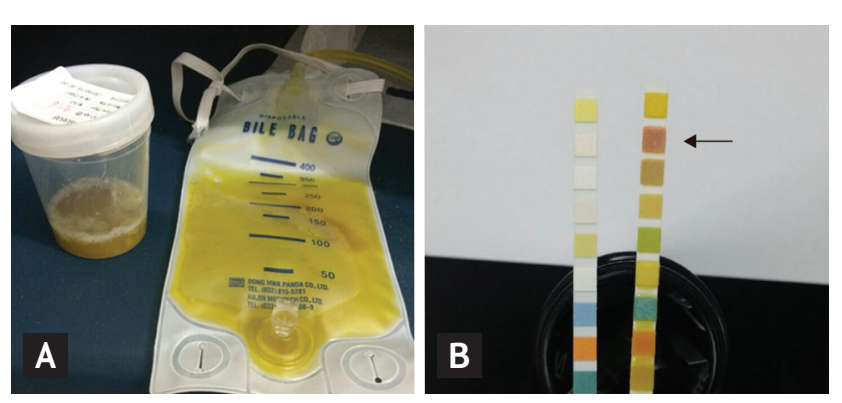

Figure 1. Biloptysis and sputum dipstick test result. (A) Brownish sputum (in sputum container). Discharge from percutaneous transhepatic biliary drainage tube (bile bag). (B) Comparison between two urinalysis strips. An unreacted strip (to the left) and a pathological (to the right, from the patient) strip. Pathological strip reacted with bilirubin in sputum and produced a characteristic color (arrow, bilirubin $[+++])$.

right lower lung and chest computed tomography (CT) revealed a consolidation in the right lower lobe with subdiaphragmatic abscess. The sputum was positive for bilirubin according to a urine dipstick test (Fig. 1). Flexible bronchoscopy showed a bile-colored secretion in the right lower lobar bronchus (Fig. 2). A tubogram was performed and revealed a dilated bile duct and a fistulous communication with the right bronchial tree (Fig. 2); thus, confirming the diagnosis of BBF. Lipiodol embolization was then performed through a PTBD tube (Fig. 3). After embolization, dramatic improvement in his bilioptysis was observed. No signs of bilioptysis were observed over 1 month of follow-up; also, his symptoms and pneumonic infiltration as assessed by chest X-rays were improved. After removal of the PTBD tube, he was discharged on the 18th day post-procedure.

In adults, BBF is a rare condition that complicates bile duct obstructions and liver abscesses. BBF can be caused by inflammatory reactions in the subdiaphragmatic space, leading to diaphragm rupture that makes the communication between the bronchial system and the biliary tract [1]. Although hydatid disease has traditionally been the leading cause of $\mathrm{BBF}$, the disease spectrum is changing. BBF has been increasingly reported to follow recent surgery or invasive interventional therapies like radiofrequency thermal ablation of hepatic tumors or TACE [1].

$\mathrm{BBF}$ is associated with a high mortality and morbidity rate (12.2\%) [1]. To prevent additional complications and fatal consequences, early diagnosis and adequate

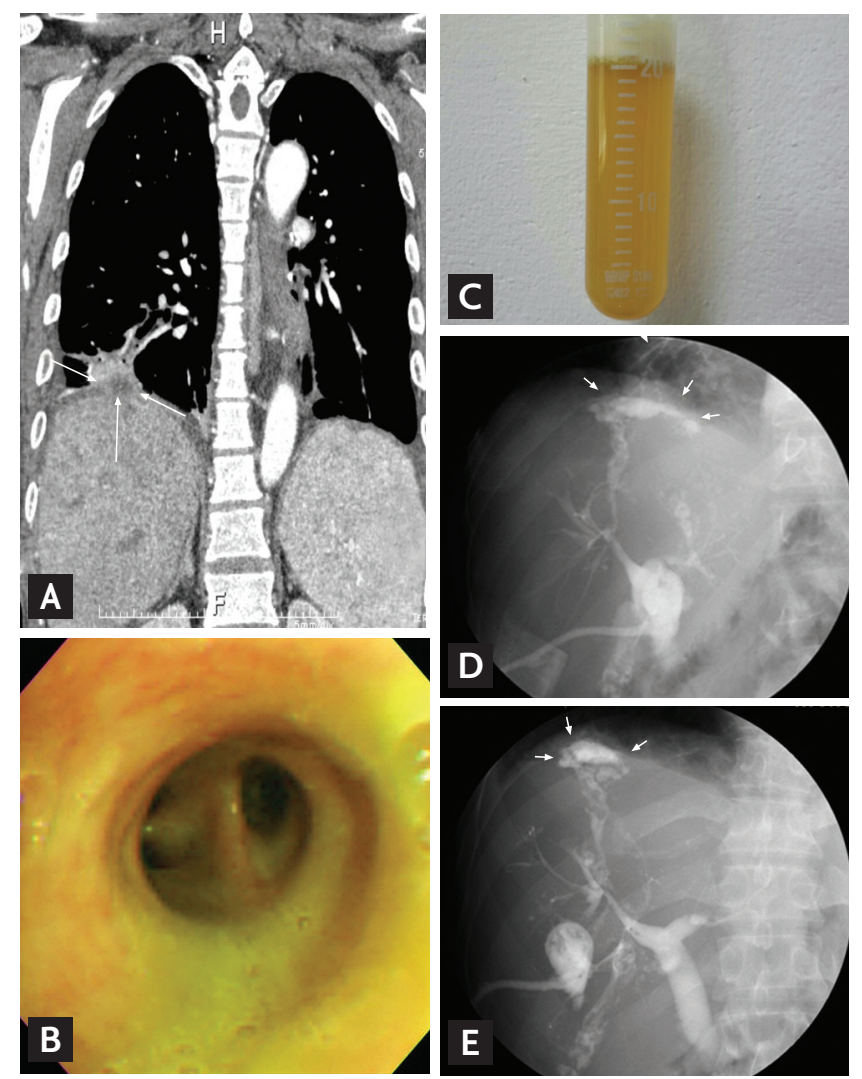

Figure 2. Bronchobiliary fistula. (A) Coronal computed tomography scan reveals a consolidation in the right lower lobe with subdiaphragmatic abscess (arrows). (B) Bile-colored secretion in right lower lobar bronchus. (C) Aspirated bronchial secretion. (D, E) Tubogram reveals dilatated bile duct and a fistulous communication with the right bronchial tree (arrows).

treatment are essential. Bile irritates the lung tissue and can also cause irritating cough, fever, and jaundice, all of which are frequent clinical features of BBF. Pneumonia is the most common comorbidity of BBF. On chest radiographs, increased pneumonic opacities on the right lower lung field are typical presentations [1]. Thoracic and upper abdominal CT scans appear to be the best tools for the initial investigation of lung and liver pathologies; this information is used to guide further treatments. Conventionally, endoscopic retrograde cholangiopancreatography (ERCP) is used to obtain direct photographic evidence. However, contrast-enhanced magnetic resonance cholangio and hepatobiliary iminodiacetic acid scanning techniques ensure definitive diagnoses and also have distinct advantages over other conventional non-invasive techniques $[2,3]$. 

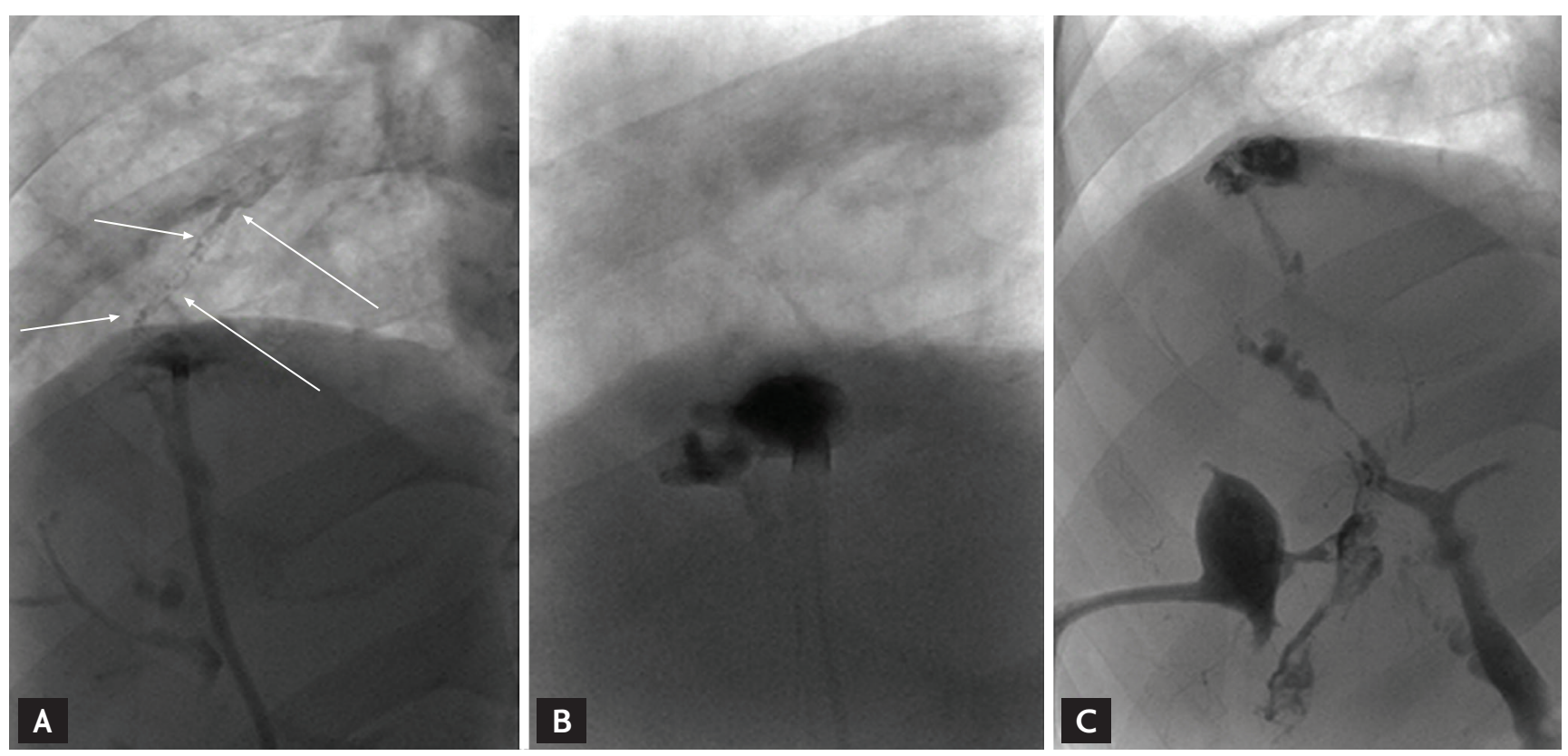

Figure 3. After diagnosed with bronchobiliary fistula: arrow in (A), the lipiodol embolization is performed (B,C).

Clinical diagnosis is usually made by the presence of bile in the sputum, referred to as biloptysis. Bile acids concentration can be quantified in sputum supernatants by an enzymatic assay (Bioquant, San Diego, CA, USA; detection limit, $0.2 \mu \mathrm{mol} / \mathrm{L}$ ) [4]. However, an easy and simple approach for detecting bilirubin in sputum is to use a urine dipstick test. This test can be performed at bedside and quickly assesses the presence of bilirubin. The bilirubin pad on the multi-reagent dipstick detects bilirubin using a diazo reagent. When diazotized sulfanilic acid reacts with bilirubin, azobilirubin is formed. This reaction is known as the Van den Bergh reaction and can be used to qualitatively estimate the amount of bilirubin present. The dipstick test can show positive results in less than one minute and is performed widely in most medical laboratories. Moreover, the test is cost effective and does not require special training to perform the test. Therefore, the urine dipstick test is potentially broadly applicable for detecting bilirubin in sputum, such as in patients with BBF.

Despite these advantages, false positive and false negative results can occur. Examples of medications that produce false positive bilirubin results include rifampin, phenazopyridine and nonsteroidal anti-inflammatory agents (etodolac, mefenamic acid). False positive reactions can be given by unproperly stored samples. Bili- rubin undergoes photo oxidation to biliverdin when it is exposed to ultraviolet light and conjugated bilirubin is hydrolyzed to unconjugated bilirubin when it is left at room temperature. The specimen should be examined within two hours of collection. Also high concentrations of vitamin $\mathrm{C}$ can produce false positive result.

Through a literature review, there was no study using the urine dipstick test to diagnose BBF. Sputum color is not sufficient to prove existing bilirubin contents in the sputum; thus, we try to apply the urine dipstick to verify the presence of bilirubin in the sputum. We suggest that the dipstick test could be used as a screening test for $\mathrm{BBF}$, and if the results of the test are positive, a confirmatory procedure would be prudent.

The definitive treatment for BBF is surgical fistulectomy with soft tissue reconstruction. However, this surgical approach is accompanied by significant morbidity and mortality, in addition to frequent reoperation. Surgery should be chosen only when interventional techniques have failed or when BBF secondary to tumors, biliary obstructions, and/or trauma is present. Less invasive methods such as external and internal stenting, which can reduce biliary obstruction, are preferred because they are much safer and easier.

Recently, embolization of fistula has been shown to be an effective treatment option for BBF [5]. Histoacryl em- 
bolization and BBF obliteration with a silicon spigot under bronchoscopic guidance have been reported as new therapeutic methods. These new methods are even easier and are associated with fewer complications than conventional less invasive intervention methods that aim to lower biliary tract pressure.

In our case, BBF developed after TACE for hepatocellular carcinoma. Despite successful percutaneous biliary dilatation and PTBD, the patient presented with persistent bile-tinged sputum and pneumonia. We performed embolization with glue and lipiodol. This case illustrates that the presence of bilirubin, which is relevant to the diagnosis of BBF, can be easily and effectively established by a sputum dipstick test. Early diagnosis followed by prompt embolization intervention can help alleviate patient suffering and may also help reduce mortality and morbidity.

Keywords: Fistula; Urinalysis; Ethiodized oil

\section{Conflict of interest}

No potential conflict of interest relevant to this article was reported.

\section{REFERENCES}

1. Liao GQ, Wang H, Zhu GY, Zhu KB, Lv FX, Tai S. Management of acquired bronchobiliary fistula: a systematic literature review of 68 cases published in 30 years. World J Gastroenterol 2011;17:3842-3849.

2. Karabulut N, Cakmak V, Kiter G. Confident diagnosis of bronchobiliary fistula using contrast-enhanced magnetic resonance cholangiography. Korean J Radiol 2010;11:493-496.

3. Annovazzi A, Viceconte G, Romano L, Sciuto R, Maini CL. Detection of a suspected bronchobiliary fistula by hepatobiliary scintigraphy. Ann Nucl Med 2008;22:641643.

4. Grabowski M, Kasran A, Seys S, et al. Pepsin and bile acids in induced sputum of chronic cough patients. Respir Med 2011;105:1257-1261.

5. Kostopanagiotou K, George RS, Kefaloyannis E, Papagiannopoulos K. Novel technique in managing bronchobiliary fistula in adults: endobronchial embolization using silicone spigots in 2 cases. Ann Thorac Med 2015;10:67-68. 